\title{
ANDERS HANSEN, DAVID MACHIN (2019). MEDIA AND COMMUNICATION RESEARCH METHODS. LONDON: RED GLOBE PRESS; 2ND ED., 314 PP., ISBN: 978-1-137-52824-7.
}

DOI: 10.51480/1899-5101.13.3(27).11

The book of Anders Hansen and David Machin provides up-to-date knowledge about research methods in media and communication studies. This is the second edition (first publish in 2013) of their work, as the authors point out in the preface:

With this second edition of Media and Communication Research Methods, we build and expand on the first edition's introduction to researching and to key approaches and methods.

The authors updated references, added new examples and expanded the discussions on selected topics. It seems reasonable in times of dynamic changes in the media landscape and communication processes, which also affect new challenges in research. Of course, research methods have generated a huge body of literature, but in this case the authors present not only methodology, but also the context of the modern communication process. The work takes up the problem of links between research methods and theory dedicated to media and communication. The authors emphasize that methods cannot be considered in isolation from theory. In addition to contemporary research methods and tools selected by the authors, there is also an attempt to indicate the roles of existing research and theoretical frameworks and that of choosing carefully and consciously methodological tools to research questions.

Media and Communication Research Methods consists of eleven chapters split into three sections. The authors begin with introductory issues dedicated to the research process, then explore selected methods and approaches, and finish on managing and analyzing data. This structure seems logical and reasonable for a book that aims to introduce key research methods and the context of changing media and communication.

The opening chapter of the first section is an Introduction, in which authors present goal of their work, describe media and communication research by presenting different approaches, pioneers of research and the development of this field. This chapter explains the links between theory and methods, as well as complementarity of quantitative and qualitative methodologies. The second 
chapter is also rather introductory and looks at the research process. The authors describe this part as an "accessible step-by-step guide to the key stages of any media and communication research project". This description best reflects the content of the second chapter. Steps of the research process are divided into eight stages from selection of a research problem to presentation and replication or further research. This important part of the book is easy to understand and well developed, because the authors created a succinct and transparent table showing all the steps, and then also describe all of them in detail.

The third chapter is dedicated to researching ownership and media policy. This part is different to subsequent chapters, because its aim is to present how to study the economy of media and specificity of media organizations. The chapter explains the role of researching ownership and control in acquiring knowledge of the nature of media organizations. In the fourth chapter, the authors present ethnography and observation methods as important elements in studies dedicated to consumption and production of media. Indeed, among the many aspects that the authors pay explore in detail, visual journalism in the context of developing observation and ethnography seems the most interesting. The section ends with practical advice on carrying out observation and ethnographic research.

The authors dedicated a substantial portion of the book, in the second section, to the different approaches to media and content. The fifth chapter focuses on content analysis. There is a brief history of this research method as well as a detailed description of the research carried out using this method. The authors divide the process of content analysis into eight steps and describe in detail all the stages from defining the research problem to reporting the results. Chapter six aims at explaining Critical Discourse Analysis (CDA) and presents the possibility of systematic analysis of text and language, the development of CDA, as well as criticism of these sets of tools. The authors introduce components of CDA and show how they can be applied in research. The seventh chapter presents analysing narratives and discourse schemas. The chapter shows the development of the approach, introduces the main terms, and explains that narrative and structural analysis provide tools for research dedicated to media genres and presents how they could be implemented. The analysis of visuals (photographs and video) is the topic of chapter eight, which is the last in the second section. The authors present the relatively late development of methodology for analysing visual communications. They also indicate a number of approaches and guidelines of how to analyse and interpret video and photographs. Again, the authors describe how to use the method in a research project.

The third and final section of the book explores and explains the use of survey research and focus group interviews. Chapter nine presents survey as frequently used for studying media audiences and other groups of people. The authors detail the numerous important aspects of this tool, like the various types of survey, their 
strengths and weaknesses, data collection, sample populations in surveys and designing questionnaires. The reader can find a similar pattern of presentation of focus group interviews in chapter ten. Semi-structured individual or group interviews are presented a having the potential to provide a richer type of data. The authors look at development, the possibilities of use, sampling, analysing and reporting in the case of interviews. They also present steps in focus group research and some examples.

Finally, the eleventh and last chapter of the book explains how to manage and analyse communication research data. This chapter presents tools to manage and analyze both qualitative and quantitative data. The chapter is divided into two parts; one dedicated to qualitative media and communication research data and the other to quantitative data. Therefore, the last part of the book illustrates a most important element of the research process and equips the reader with practical knowledge about analyzing data. This chapter seems appropriate to complete the book on media and communication research methods.

Without doubt, the book by Anders Hansen and David Machin is a valuable compendium of knowledge on research methods in the field of media and communication. The structure of work is logical and transparent. At the beginning the authors introduce general knowledge of the research process, then describe selected approaches and methods in the following parts of the book and at the end give readers practical knowledge about analyzing data. In the chapters dedicated to methods and approaches, the authors present short summaries and recommend further readings. The book would be helpful for students who wish to learn about methodology in media and communication studies. In general, this work could be seen to be a response to the needs of students and could be valuable resource for methodology courses. The book contains up-to-date knowledge about research methods in media and communication studies, which could all, but especially young, researchers to create their own projects. This work is a successful attempt to create a helpful guide to the main methods and stages of media and communication research.

Paulina Barczyszyn-Madziarz UNIVERSITY OF WROCŁAW 